of Bruch's membrane at the optic nerve. Three cases of partial cross ruptures of the optic nerve are reported histologically. All three are discovered at the very insertion of the lamina elastica chorioideae. The tear was caused in all cases (traumatic, expulsive haemorrhage and haemorrhagic glaucoma) by an instantaneous, probably arterial, subretinal bleeding. Traumatic retropulsion of the optic nerve or propulsion of the bulb causes cross ruptures of the optic nerve, too, occurring at the same spot and leading to evulsion of the optic nerve.

\title{
REFERENCES
}

Ballantyne.-Mackenzie Memorial Lecture: Vascular Disease and its Significance in Medical Ophthalmology. Glas. Med. Jl., Vol. XX, p. 26, 1942.

Lagrange, F.-Atlas d'Ophtal. de Guerre, Masson, Paris, 1918.

VON Michel.-Zeitschr.f. Augenheilk., Vol. VI, p. 1, 1901.

Salzmann.-Zeitschr. f. Augenheilk., Vol. IX, p. 489, 1903.

VoN SzILY.-Atlas der Kriegsaugenheilkunde. F. Enke, Stuttgart, 1918.

Wagenmann.-Verletzungen des Auges. Graefe Saemisch-Handbuch der Augenheilkunde, 3rd Ed., II, Chap. 17, 1915.

WÜrdeMANN, H. V.-Injuries of the Eye. London, 2nd Ed., 1932.

\section{ORTHOPTIC TREATMENT OF ANOMALOUS PROJECTION}

\author{
BY
}

Diana S. MANN, B.SC.

MELBOURNE

While theory concerning the nature of abnormal retinal correspondence or anomalous projection has been considerably developed in the last ten years, notably by the writings of Chavasse, Verhoeff and Travers, practice in its treatment has hardly kept pace. In this paper an attempt is made to outline briefly the theoretical conceptions which prove to be of most practical value in guiding an extension of training methods. Of the many names under which the phenomenon goes, Verhoeff's term, "anomalous projection,". is adopted as being most in accordance with these conceptions.

Ordinary binocular vision, in which both visual axes are always directed on the same object by reflex fusion mechanism, is an innate habit, for although it develops after birth, it does so along predisposed lines. The same applies to the mental processes that interpret the two images received, one by each macula, as being a single object (if the images are similar), or as representing two 
objects in the same place (if the two images are dissimilar). A further innate habit is suppression of one macular image when they are dissimilar, as often happens when a distant object is hidden from one eye by a nearer object; while one eye is fixed on the far object, the image of the nearer. falls on the macula of the other eye, but in most cases it is only. by careful attention that one can see it. In squint the faculty of suppression may be developed simply by constant use, and it being part of the innate visual mechanism, this is done without difficulty.

On the other hand, those who react to squint by developing what is known as abnormal correspondence or anomalous projection, do so by maintaining a constantly high level of attention on the two unlike macular scenes so as to keep one separated from the other, and to project each approximately to its true position. Anomalous projection requires a high level of attention because it is not an innate tendency, but is an abnormal use of the visual nervous mechanism ; it may become habitual, but imposes a greater nervous strain than the normal reflex of suppression. Suppression of one macular field in squint is developed by concentrating attention on the other conflicting field. A direct means of attacking suppression, therefore, is to excite attention in the previously neglected direction. An equally direct means of attacking anomalous projection is harder to find, because divided attention between two conflicting macular fields is probably influential in its genesis. One finds that in anomalous projection of recent origin, a pair of ùnlike pictures, such as used for testing S.M.P. on the synoptophore, will be perceived as widely separated when placed one in front of each eye, while a fusible pair of pictures may be not only fused, but seen stereoscopically.

Practising such fusion may be used as the first step in the recovery of normal vision. It cannot, it is important to remember, be used to straighten the eyes in a convergent squint at this stage since, if abduction is attempted, the eyes fail to maintain fusion and can be seen to move as the patient looks from one picture to the other, though he will insist that he sees only one. Presumably. by producing homonymous diplopia, one has imitated the conditions under which anomalous projection was developed, so that the patient's mind reverts to this state. However, the response to attempted adduction is far better and, in convergent squints, one may develop some range of fusion (between the resting angle and a more convergent one) by this means; the patient is aware of diplopia when convergence fails. Even in divergent squints, if of the type where ability to converge on near objects is not lost, the patient may maintain genuine fusion by convergence. He will not be aware of diplopia when fusion breaks, but will notice loss of stereosscopic depth there. 
The possibility of fusion and stereopsis without normal S.M.P.* is important in regard to the treatment of anomalous projection, but two other facts are equally so. One is that the disruption of normal correspondence does not apply over the whole binocular field. This is shown by the apparent behaviour of images when synoptophore tubes are moved from zero towards the angle of squint. If one eye, let us say the right, be fixed on a lion, the image of the cage will fall on an eccentric part of the left retina, which normally receives the duplicate (which is disregarded) of what the right eye is fixing. Once attention is aroused to this cage, it is perceived as lying to the left of the lion; and if attention and fixation are firmly centred on the lion, and the cage is moved steadily towards the angle of squint, the cage is seen to move towards the lion and (in a fairly recent anomalous projection) momentarily surrounds it before it "jumps" away to the right. The apparent behaviour previous to the jump is exactly that which would occur under similar conditions in normal projection, and is as if the cage image fell on the normally corresponding points in the eye that is fixed on the lion. The jump occurs when the cage image falls on a part of the left retina, i.e., the macula, where the attention level is sufficiently high for it to be recognised as a left retinal image, and therefore to be projected to the right side of the lion. The jump occurs sooner in an old-established anomalous projection, where a high attention level has spread to a greater distance from the macula; and when steady monocular fixation is not maintained it occurs much earlier still-usually when the cage image reaches a point about $15^{\circ}$ from the angle of (convergent) squint, for the disappearance of the non-fixed image as it falls on the blind spot induces an immediate movement to fix it with the macula, which projects it accurately with respect to the image seen a moment before with the other macula.

When the tubes are moved from the angle of squint towards zero, the "jump" back occurs at a point nearer zero than the " jump" in the opposite direction, as once the individual projection of each retina is excited, normal projection reasserts itself with more difficulty.

The third point is that the apparent " abnormal correspondence" of the macula of one eye with a point some $15^{\circ}$ from the macula of the other eye hardly exists until it has been aroused by tests and measurements. A frequent description of the pictures as seen with two eyes is that they seem to be " in different worlds," and the indecision of the patient whose prolonged efforts to "put the lion in the cage" may ultimately be abandoned as impossible;

- S.M.P. is here used for the sake of brevity in the generally accepted sense, as implying normal macular correspondence in the presence of unlike macular images as well as simultaneous perception. 
or by fixing on a position where "I can't see them both properly, but it must be in the cage somewhere about here," indicates that he is governed by no direct sense of their relative projection, but rather is trying to reconcile two conflicting impressions. The apparent illogical failure of his own senses inevitably excites the interest of the patient, whatever his age, and if allowed he will make repeated attempts to cage the lion by movements from either side, and to convince himself that in some position they coincide. Not only does this help to settle the abnormal relationship more firmly in his mind, but it encourages an analytical attentive mental attitude; whereas normal correspondence is best regained by relaxing mental vigilance to give the innate nervous mechanism a chance. It is, on the whole, unwise even to measure the angle of anomaly, as this -may start a false train of thought in the patient's mind.

\section{Treatment of Anomalous Projection-Pre-Operative.}

1. Maintain constant total occlusion of one eye except during orthoptic exercise, so that the need for independent projection disappears.

2. Do not measure the angle of anomaly except in special cases, when one should indicate by one's manner that the results are unimportant.

3. During treatment the patient must watch the pictures constantly, but for most exercises will do best if his attention is distracted by stories, conversation about home affairs, discussion of some detail in the appearance of the pictures, or any subject other than the relative position of the two pictures before him. To maintain the right state of attentive inattention is the most difficult and most important part of the orthoptist's work. Each patient will require different methods of approach, and will respond best to different combinations of the methods outlined in 4 and 5 .

4. Normal correspondence may be encouraged most readily by inserting fusion pictures, preferably. with fusion check and some stereoscopic element at the angle of squint, locking the tubes and (1) shaking them in front of the patient in a quick jerky manner, (2) rapidly alternating the lights behind the pictures, (3) making the patient shut his eyes for a moment and then look again. Once a single image is perceived and held, attention to check marks and stereopsis is encouraged. When fusion can be maintåned in the primary position, adduction (in convergent squints) or abduction (divergents) should be developed, to encourage fusion reflex movements without risk of falling back on abnormal correspondence.

5. Normal correspondence is not firmly established until S.M.P. slides are seen together at the true angle of squint. Methods of achieving this are :- 
(a) The patient fixes the lion while the cage is swept up steadily from outside the angle. It is important that fixation should be steady, the story being that " the lion will get frightened if he knows the cage is coming, so we must pretend not to know it's there." The cage tube should be carried on a little past the angle of squint, and then moved backwards and forwards for a while across it, as long as the cage seems to be passing across the lion. The subsequent return movement of the tube to the outward position must be done quickly while talking so that the reverse approach of the cage is disregarded. If the patient takes an interest in the return movement, or if he fails to maintain steady fixation on the lion, this exercise must be abandoned.

'(b) The patient maintains fixation on the lion, while the cage tube is moved backwards and forwards across the angle.

(c) The tubes are locked together at the angle and shaken.

(d) If the patient is intelligent, one may carry on a modified form of "chasing.". First it is demonstrated to him that at the true angle the pictures are both seen at once more clearly than in any other position (although they appear to be separated in space). The orthoptist then moves one of the tubes, and the patient moves the other so as to bring the pictures again to the clearest position. This exercise must, of course, be abandoned if the patient attempts to approximate the pictures at the "false" angle.

In all of the above one may, of course, use any other pair of S.M.P. slides, using very large angle ones at first, and finally the smallest.

6. When all traces of anomalous projection with S.M.P. pictures has disappeared, and some range of duction has been developed as in (3), reduction of the squint by abduction (convergent squints) or adduction (divergent) may be attempted. Constant vigilance, however, is necessary to make sure that the patient is not suppressing and returning to anomalous projection.

7. Operation is almost inevitably necessary, and there is no point in prolonging treatment before operation if the patient shows no improvement in three or four visits. The only general prerequisites are that the vision should be equal and the patient should be able to alternate readily. When time is short, as with country patients, operation should be performed as soon as this state is attained. The choice of operation will be affected by the following considerations :-

(a) Operative reduction or correction of the squint inevitably leads, in time, to reduction of the angle of anomaly, and often restores normal correspondence surprisingly quickly, or speeds up the response to orthoptic methods. 
(b) A good operative result (angle 0 to $-0^{\circ}$ ) after convergent squint obviates the necessity for developing S:M.P., as this is not required as a pre-requisite for adductions, but may develop automatically as the fusion range increases. "Adduction improves rapidly with convergence exercises on near objects; as fusion, however weak, is then reinforced by accommodation-convergence.

(c) A post-operative convergence after convergent squint, on the other hand, is difficult to treat unless good S.M.P. and fusion amplitude are present. If there is any tendency to suppression, this will increase rapidly to counteract diplopia in the new position.

(d) Certain cases, much quoted in text-books but fortunately rare, tend to recur after operation. This may possibly be due to annoyance caused by inaccurate post-operative projection and diplopia, but may happen even when anomalous projection has disappeared. Recurrence is most likely. to take place when the angle before operation is variable with a considerable accommodative element, and the patient is of a nervous and excitable type. Although it would appear that a special effort should be made to obtain normal correspondence before operation in such cases, they are usually particularly stubborn ones; the long training may have the effect of concentrating attention on the eyes, so that any nervous excitement acts as a direct stimulus to convergence, and the squint may recur in spite of recovery of normal correspondence. The question is complicated and is probably one in which use of sedatives and attention to psychic and home influence is of more importance than orthoptics.

8. Post-Operative.-When the operative result is good, the probability is that some binocular vision may be recovered in time without orthoptic treatment. However, the recovery is seldom complete, and the patient may develop typical eye-strain symptoms at once or years afterwards which clear up when a good fusion range is developed. If symptoms do not develop or are not combated until some years after operation, habits of suppression may by this time have grown too strong to be overcome, and one is unable to relieve the symptoms. It is therefore best to give orthoptic treatment until a range of fusion of at least $+25^{\circ}$ to $0^{\circ}$ with some stereopsis is obtained.

Treatment is essentially the same as pre-operative, making allowance for the altered angle of squint with consequent change in position of the "false correspondence " angle. Exceptions are in post-operative divergence after convergent squint. First, occlusion is contra-indicated for such cases unless there is very 
marked suppression and divergence. Secondly, as there is no need to increase power of abduction; it is unnecessary to insist on S.M.P., though this is, of course, an advantage. The power of convergence may be greatly developed by practice on a pencil, etc., as for convergence deficiency, and one often finds that S.M.P. is attained readily after such a stimulus to normal binocular vision.

Should the abnormal correspondence not yield readily to treatment after operation, the angle being satisfactory, it is probably wisest to leave the case alone, with observation at intervals, so that when time has done its bit in breaking down the abnormal correspondence, one may jump in and deal the death blow.

Although the treatment as here indicated includes some timehonoured methods, the omission of several useful tricks will be obvious to orthoptists. My aim has been to make a statement as simple as possible.

\title{
CONTACT LENS IN CASES OF NEUROPARALYTIC KERATITIS
}

\author{
BY - \\ M. KLEIN \\ , LONDON
}

AMONG the indications for the use of contact lenses neuroparalytic keratitis does not seem to have attracted much attention. This record of their use in two cases may therefore be of interest.

1. Miss V. P., aged 39 years. Had several operations for right sided acoustic tumour. The last operation left her with a complete right facial palsy and trigeminal anaesthesia. Neuroparalytic keratitis of the right eye developed, which eventually made tarsorraphy necessary, but in spite of this, the state of the cornea did not improve. After a year, though the lids were still sutured, the condition of the eye seemed hopeless. The therapeutic effect of a contact lens was then tried as a last resort (August, 1942). Rapid improvement followed, and withih 2-3 weeks the eye became white, the cornea cleared up considerably, except at the central area where a dense scar remained. The patient is wearing the contact lens regularly from 7 a.m. to 1 p.m. when it is removed for about halfan-hour, and generally worn again till about 9 p.m.

An incident emphasised the value of the contadt lens. In November, 1942, the patient accidentally broke the contact lens. The eye became inflamed within a few hours, and by the following

From the Central London Ophthalmic Hospital. 